TRANSACTIONS OF THE

AMERICAL MATHEMATICAL SOCIETY

Volume 181, July 1973

\title{
A LAW OF ITERATED LOGARITHM FOR STATIONARY GAUSSIAN PROCESSES
}

\author{
BY \\ PRAMOD K. PATHAK AND CLIFFORD QUALLS
}

ABSTRACT. In this article the following results are established.

Theorem A. Let $\{X(t): 0 \leq t<\infty\}$ be a stationary Gaussian process with continuous sample functions and $E[X(t)] \equiv 0$. Suppose that the covariance function $r(t)$ satisfies the following conditions.

(a) $r(t)=1-|t|^{a} H(t)+o\left(|t|^{\alpha} H(t)\right)$ as $t \rightarrow 0$, where $0<a \leq 2$ and $H$ varies slowly at zero, and

(b) $r(t)=O(1 / \log t)$ as $t \rightarrow \infty$.

Then for any nondecreasing positive function $\phi(t)$ defined on $[a, \infty)$ with $\phi(\infty)=\infty, P\left[X(t)>\phi(t)\right.$ i.o. for some sequence $\left.t_{n} \rightarrow \infty\right]=0$ or 1 according as the integral $I(\phi)=\int_{a}^{\infty} g(\phi(t)) \phi(t)^{-1} \exp \left(-\phi^{2}(t) / 2\right) d t$ is finite or infinite, where $g(x)=1 / \tilde{\sigma}^{-1}(1 / x)$ is a regularly varying function with exponent $2 / \alpha$ and $\tilde{\sigma}^{2}(t)=2|t|^{\alpha} H(t)$.

Theorem C. Let $\left\{X_{n}: n \geq 1\right\}$ be a stationary Gaussian sequence with zero mean and unit variance. Suppose that its covariance function satisfies, for some $\gamma>0, r(n)=O\left(1 / n^{\gamma}\right)$ as $n \rightarrow \infty$. Let $\{\phi(n): n \geq 1\}$ be a nondecreasing sequence of positive numbers with $\lim _{n \rightarrow \infty} \phi(n)=\infty$; suppose that $\Sigma(1 / \phi(n)) \exp \left(-\phi^{2}(n) / 2\right)=\infty$. Then

$$
\lim _{n \rightarrow \infty} \sum_{1 \leq k \leq n} I_{k} / \sum_{1 \leq k \leq n} E\left[I_{k}\right]=1 \quad \text { a.s., }
$$

where $I_{k}$ denotes the indicator function of the event $\left\{X_{k}>\phi(k)\right\}$.

1. Introduction. Let $\left\{S_{n}: n \geq 1\right\}$ denote the sequence of partial sums of independent and identically distributed Gaussian random variables. The classical law of iterated $\log$ arithm states that $\lim \sup _{n} s_{n} / \sqrt{2 s_{n}^{2} \log \log s_{n}^{2}}=1$ a.s., where $s_{n}^{2}=\operatorname{Var}\left(s_{n}\right)$. Feller's generalization and the "best possible" result [1], [2], when applied to this case states that for any nondecreasing sequence of positive numbers $\left\{\phi_{n}: n \geq 1\right\}$ with $\lim \phi_{n}=\infty, P\left[s_{n}>\phi_{n} s_{n}\right.$ i.o. $]=0$ or 1 according as $\Sigma\left(\phi_{n} / n\right) \exp \left(-\phi_{n}^{2} / 2\right)<\infty$ or $=\infty$. (The classical law of iterated logarithm follows trivially from Feller's result.) During the past five years, Pickands [4], Qualls

Received by the editors June 19, 1972.

AMS (MOS) subject class ifications (1970). Primary 60F10, 60F20, 60G10, 60G15, 60G17.

Key words and phrases. Stationary process, Gaussian process, law of iterated logarithm, zero-one law, upcrossings, rate of growth of upcrossings. 
and Watanabe [7], [8], Qualls, Simmons and Watanabe [9], and others have obtained a number of Feller-type results for stationary Gaussian processes. The main object of this paper is to make a careful study of these results and to show that some of these results are valid under weaker conditions. We also propose certain new lines of investigation. An outline of our results is as follows.

Theorem A. Let $\{X(t): 0 \leq t<\infty\}$ be a stationary Gaussian process with continuous sample functions and $E[X(t)] \equiv 0$. Suppose that the covariance function $r(t)$ satisfies the following conditions:

(a) $r(t)=1-|t|^{\alpha} H(t)+o\left(|t|^{\alpha} H(t)\right)$ as $t \rightarrow 0$, for some $\alpha$ with $0<\alpha \leq 2$ and some function $H(t)$ which is slowly varying at zero, and

(b) $r(t)=O(1 / \log t)$ as $t \rightarrow \infty$.

Then for any nondecreasing positive function $\phi(t)$ defined on $[a, \infty)$ with $\phi(\infty)=$ $\infty, P\left[X(t)>\phi(t)\right.$ i.o. for some sequence $\left.t_{n} \rightarrow \infty\right]=0$ or 1 according as the integral $I(\phi)=\int_{a}^{\infty} g(\phi(t)) \phi(t)^{-1} \exp \left(-\phi^{2}(t) / 2\right) d t$ is finite or infinite where $g(x)=$ $1 / \widetilde{\sigma}^{-1}(1 / x)$ is a regularly varying function with exponent $2 / \alpha$ and $\widetilde{\sigma}^{2}(t)=$ $2|t|^{a} H(t)$.

That the probability of the event considered above is zero or one is not surprising. It is easily seen (see [9]) that the mixing condition: $\lim _{t \rightarrow \infty} r(t)=0$ is sufficient for the zero-one law. In fact the ergodic condition: $\lim _{T \rightarrow \infty}(1 / T) \int_{0}^{T} r(t) d t=0$ would suffice.

Theorem A was first established by Watanabe [11] under the stronger mixing condition: $r(t)=O(1 / t)$ as $t \rightarrow \infty$. Later Watanabe and Qualls [7] showed that the theorem remains valid under the weaker mixing condition: $r(t)=O\left(1 / t^{\gamma}\right)$ as $t \rightarrow \infty$. In this paper, the Paley-Zygmund lemma, Lemma 2.4 below, allows us to attain a still weaker mixing condition.

Theorem $B$, which appears in $\$ 3$, furnishes an analogous result for discrete parameter stationary Gaussian processes. This theorem strengthens similar results due to Pickands [4] and Qualls, Simmons and Watanabe [9].

It is perhaps worth mentioning that similar improvements are possible for corresponding results in random fields (see Qualls and Watanabe [10]) and for certain nonstationary processes (see Qualls and Watanabe [8]).

In $\$ 4$, we propose a new line of investigation and establish the following sharper results in the discrete parameter case:

Theorem C. Let $\left\{X_{n}: n \geq 1\right\}$ be a stationary Gaussian sequence with zero mean and unit variance. Suppose that its covariance function satisfies, for some $\gamma>0$,

(b) $r(n)=O\left(1 / n^{\gamma}\right)$ as $n \rightarrow \infty$.

Let $\{\phi(n): n \geq 1\}$ be a nondecreasing sequence of positive numbers with $\lim _{n \rightarrow \infty} \phi(n)=\infty$ and suppose that $\Sigma(1 / \phi(n)) \exp \left(-\phi^{2}(n) / 2\right)=\infty$. Then 


$$
\lim _{n \rightarrow \infty} \sum_{1 \leq k \leq n} I_{k} / \sum_{1 \leq k \leq n} E\left[I_{k}\right]=1 \text { a.s. }
$$

where $I_{k}$ denotes the indicator function of the event $\left\{X_{k}>\phi(k)\right\}$.

2. Proof of Theorem 1. We refer the reader to Qualls and Watanabe [8] for the proof of the theorem when $I(\phi)<\infty$. For the crucial second half of the proof, we need the following lemma from [8]:

Lemma 2.1. If condition (a) of Theorem A bolds and $r(s) \neq 1$ for $0<s<t$ then for any $a>0$

$$
\lim _{x \rightarrow \infty} \frac{P\left[\max _{0 \leq k \leq m} X(k a / g(x))>x\right]}{t(2 \pi)^{-12} g(x) x^{-1} \exp \left(-x^{2} / 2\right)}=\frac{H_{a}(a)}{a}
$$

where $m=[\operatorname{tg}(t) / a]$ with [] denoting the greatest integer function, and $H_{\alpha}(a)$ is a certain positive constant. Note that $\tilde{\sigma}^{2}(t)=2|t|^{\alpha} H(t)$ is monotone near zero and that $\tilde{\sigma}^{2}(t) \sim \sigma^{2}(t)$ as $t \rightarrow 0$, where $\sigma^{2}(t)=E(X(t)-X(0))^{2}=2(1-r(t))$, and recall the notation $g(x)=1 / \tilde{\sigma}^{-1}(1 / x)$.

We also need the following lemmas from [7] and [8]:

Lemma 2.2. Let $I(\phi)=\infty$ and suppose that the second part of Theorem $A$ bolds under the following added restriction: for large $t$

$$
2 \log t \leq \phi^{2}(t) \leq 2 \log t+2 A \log \log t,
$$

where $A>1 / 2+1 / \alpha$. Then the theorem holds without this restriction. Then

Lemma 2.3. Let $E_{n}=\left\{X\left(t_{n, \nu}\right) \geq x_{n, \nu}: \nu=0, \cdots, m_{n}\right\}$ with all $t_{n, \nu}$ distinct.

$$
\left|P\left(E_{i} \cap E_{j}\right)-P\left(E_{i}\right) P\left(E_{j}\right)\right| \leq \sum_{\mu=0}^{m_{j}} \sum_{\nu=0}^{m_{i}}|r| \int_{0}^{1} n\left(x_{i, \nu}, x_{j, \nu} ; \lambda r\right) d r
$$

where $n(x, y ; \lambda r)$ is the standard bivariate normal density with correlation coefficient $\lambda r=\lambda r\left(t_{j, \mu}-t_{j, \nu}\right)$. This result is also true for the events, $E_{n}^{c}$.

We shall also need the following lemmas:

Lemma 2.4 (Paley-Zygmund). Let $X \geq 0$ be a random variable satisfying $E\left[X^{2}\right] \leq B(E[X])^{2}<\infty$. Then $P[X>\alpha E(X)] \geq(1-\alpha)^{2} / B$, for $0<\alpha<1$. (See Zygmund [12, Lemma 8.2.6, p. 216].)

Lemma 2.5. Let $\left\{I_{n}: n \geq 1\right\}$ be a sequence of indicator functions such that $\Sigma_{1}^{\infty} E\left[I_{k}\right]=\infty$. If for some $A, 0 \leq A<\infty, \operatorname{Var}\left(\Sigma_{1}^{\infty} I_{k}\right) \leq A\left(\Sigma_{1}^{n} E\left[I_{k}\right]\right)^{2}$ for all sufficiently large $n$ then $P\left[\Sigma_{1}^{\infty} I_{k}=\infty\right] \geq 1 /(1+A)$.

Proof. Let $J_{n}=\Sigma_{1 \leq k \leq n} I_{k}$. Then $E\left[J_{n}^{2}\right] \leq(1+A)\left(E\left[J_{n}\right]\right)^{2}$. Lemma 2.4 now 
yields $P\left[J_{n}>a E\left[J_{n}\right]\right] \geq(1-\alpha)^{2} /(1+A)$ for $0<a<1$ and for large $n$. Since $\lim E\left[J_{n}\right]=\infty, P\left[\Sigma_{1}^{\infty} I_{k}=\infty\right] \geq(1-\alpha)^{2} /(1+A)$ for $0<\alpha<1$.

With these lemmas we can now furnish the proof of Theorem $A$ when $I(\phi)=\infty$.

Proof of Theorem $\mathrm{A}$ where $I(\phi)=\infty$. Consider a sequence of intervals $[n \Delta$, $n \Delta+\beta]$ with $\Delta>0$ and $0<\beta<\Delta$. For arbitrary $a>0$, let $G_{k}=\left\{t_{k, \nu}=k \Delta+\right.$ $\left.\nu a / n_{k}: \nu=0, \cdots,\left[\beta n_{k} / a\right]\right\}$, where $n_{k}=[g(\phi(k \Delta+\beta))]$. Let $E_{k}=\left[\max _{s \in G_{k}} X(s)>\right.$ $\phi(k \Delta+\beta)]$ and $I_{k}=I_{E_{k}}$ and consider partial sums, $\Sigma_{m \leq k \leq n} I_{k}$, of the se indicator functions. Now Lemma 2.1 implies that there is a positive $K$ such that

$$
E\left[I_{k}\right] \sim K \beta g(\phi(k \Delta+\beta)) \phi(k \Delta+\beta)^{-1} \exp \left\{-\phi^{2}(k \Delta+\beta) / 2\right\}
$$

as $\phi(k \Delta+\beta) \rightarrow \infty$. To see that the hypotheses of Lemma 2.1 are satisfied, note that condition (b) of Theorem A excludes periodic $r(t)$. Consequently

$$
\infty=I(\phi) \leq \sum_{m \leq k<\infty}(2 \pi)^{-1} g(\phi(k \Delta)) \phi(k \Delta)^{-1} \exp \left\{-\phi^{2}(k \Delta) / 2\right\} \cdot \Delta
$$

implies

$$
\sum_{m \leq k<\infty} E\left[I_{k}\right]=\infty
$$

It is also easily seen that

$$
\sum_{m \leq k \leq n} E\left[I_{k}\right] \geq\left(K_{1} / \Delta\right) \int_{m \Delta}^{n \Delta} g(\phi(t)) \phi(t)^{-1} \exp \left(-\phi^{2}\right) / 2 d t .
$$

That the integral $I(\phi)$ can be estimated by the above sums follows from its integrand being monotone for $m$ sufficiently large. Denoting $\phi(i \Delta+\beta)=\phi_{i}$, we obtain, via Lemma 2.3,

$$
\begin{aligned}
& \operatorname{Var}\left(\sum_{m}^{n} I_{k}\right)=2 \sum_{m \leq i<j \leq n}\left(E I_{j} I_{j}-E I_{i} E I_{j}\right)+\sum_{m \leq k \leq n}\left(E I_{k}-\left(E I_{k}\right)^{2}\right) \\
& \quad=2 \sum_{m \leq i<j \leq n} \sum_{\mu=0}^{\left[\beta n_{i} / a\right]} \sum_{\nu=0}^{\left[\beta n_{j} / a\right]}|r| \int_{0}^{1} n\left(\phi_{i}, \phi_{j} ; \lambda r\right) d \lambda+o\left(\sum_{m}^{n} E I_{k}\right)^{2},
\end{aligned}
$$

where $r=r\left(t_{j, \nu}-t_{i, \mu}\right)$. Because $t_{j, \nu}-t_{i, \mu} \geq j \Delta-i \Delta-\beta \geq \Delta-\beta$ and because of condition (b) of Theorem $A$, we have, for $\Delta$ large enough,

$$
\left|r\left(t_{j, \nu}-t_{i, \mu}\right)\right| \leq M(2 \log (j \Delta-i \Delta-\beta))^{-1} \leq \delta / 3<\delta / 2 .
$$

Consequently 


$$
\begin{gathered}
\operatorname{Var}\left(\sum_{m \leq k \leq n} I_{k}\right) \leq \sum_{m \leq i<j \leq n} \frac{\beta^{2}}{a^{2}} g\left(\phi_{i}\right) g\left(\phi_{j}\right) \cdot \frac{M(2 \pi)^{-1}\left(1-\delta^{2}\right)^{-1 / 2}}{\log (j \Delta-i \Delta-\beta)} \\
\cdot \exp \left\{-\left(\phi_{i}^{2}+\phi_{j}^{2}-2|r| \phi_{i} \phi_{j}\right) / 2\right\}+o\left(\sum_{m}^{n} E I_{k}\right)^{2} \\
\leq K_{2} M \sum_{i=m}^{(n-1)} \sum_{k=1}^{n-i} \frac{g\left(\phi_{i}\right) g\left(\phi_{i+k}\right)}{\log (k \Delta-\beta)} e^{-\phi_{i}^{2} / 2-\phi_{i+k}^{2} / 2}
\end{gathered}
$$

$$
\begin{gathered}
\cdot \exp \left\{\frac{M \phi_{i} \phi_{i+k}}{2 \log (k \Delta-\beta)}\right\}+o\left(\sum_{m}^{n} E I_{k}\right)^{2} \\
\leq \frac{K_{2} M^{\prime}}{\Delta^{2}} \int_{m \Delta}^{z-\Delta} \int_{\Delta}^{z-s} \frac{g(\phi(s)) g(\phi(s+t))}{\log t} \cdot \exp \left\{-\left(\phi^{2}(s)+\phi^{2}(s+t)\right) / 2\right\} \\
\cdot \exp \left\{\frac{M^{\prime} \phi(s) \phi(s+t)}{2 \log t}\right\} d t d s+o\left(\sum_{m}^{n} E I_{k}\right)^{2}
\end{gathered}
$$

where $z$ is a continuous variable with $n=[z / \Delta], M^{\prime}$ is arbitrarily close to $M$, and $M^{\prime}>M$.

Consider the ratio

$$
\frac{\operatorname{Var}\left(\sum_{m}^{n} I_{k}\right)}{\left(\sum_{m}^{n} E I_{k}\right)^{2}} \leq \frac{K_{2} M^{\prime}}{K_{1}^{2}} \frac{\int_{m \Delta}^{z-\Delta} \int_{\Delta_{-} \beta}^{z-s} f(s, t) d t d s}{\left(\int_{m \Delta}^{z} g(\phi(t)) \phi(t)^{-1} \exp \left(-\phi^{2}(t) / 2\right) d t\right)^{2}}+o(1)
$$

as $n=[z / \Delta] \rightarrow \infty$, where the integrand $f(s, t)$ appears in the last line of (2.2).

Using L'Hospital's rule in (2.3), we obtain

$$
\frac{\operatorname{Var}\left(\sum_{m}^{n} I_{k}\right)}{\left(\sum_{m}^{n} E I_{k}\right)^{2}} \leq \frac{K_{2} M^{\prime}}{K_{1}^{2}} \frac{\int_{m \Delta}^{z-\Delta} f(s, z-s) d s}{2 g(\phi(z)) \phi(z)^{-1} \exp \left(-\phi^{2}(z) / 2\right) I(\phi(z))}+o(1)
$$

$$
\begin{aligned}
& =\frac{K_{2}}{K_{1}^{2}}\left(\int_{m \Delta}^{z-\Delta} g(\phi(s)) \phi(s)^{-1} e^{-\phi^{2}(s) / 2} \frac{M^{\prime} \phi(s) \phi(z)}{2 \log (z-s)} \exp \left\{\frac{M^{\prime} \phi(s) \phi(z)}{2 \log (z-s)}\right\} d s\right) / I(\phi(z)) \\
& \quad+o(1)
\end{aligned}
$$

as $n \rightarrow \infty$, where $I(\phi(z))=\int_{m \Delta}^{z} g(\phi(t)) \phi(t)^{-1} e^{-\phi^{2}(t) / 2} d t$. Note that the new $z$ in (2.4) is not the same as the ' $z$ ' in (2.3).

Divide the integral in the numerator of (2.4) into two parts according as $m \Delta<s \leq \theta_{z} z-\Delta$ or $\theta_{z} z-\Delta<s<z-\Delta$, where $1-\theta_{z}=z^{-\gamma}(\log z)^{-1}$ and $\delta<\gamma<1$. The integral from $\theta_{z} z-\Delta$ to $z-\Delta$ can be ignored since we can replace $M^{\prime} / \log (z-s)$ by $\delta$ and bound this part of the integral by

$$
\left(z-\theta_{z} z\right) g\left(\phi\left(\theta_{z} z-\Delta\right)\right) \phi\left(\theta_{z} z-\Delta\right)^{-1} \exp \left\{-\phi^{2}\left(\theta_{z} z-\Delta\right) / 2\right\} \delta(\log z) z^{\delta} .
$$


This contribution to the integral in the numerator of (2.4) divided by a lower estimate of the denominator leaves the ratio $\left(1-\theta_{z}\right) z^{\delta} \log z=z^{\delta-\gamma}$ which goes to zero as $z$ goes to $\infty$. For $s \leq \theta_{z} z-1$,

$$
\frac{M^{\prime} \phi(s) \phi(z)}{2 \log (z-s)} \exp \left\{\frac{M^{\prime} \phi(s) \phi(z)}{2 \log (z-s)}\right\} \leq \frac{M^{\prime} \phi^{2}(z)}{2 \log z\left(1-\theta_{z}\right)} \exp \left\{\frac{M^{\prime} \phi^{2}(z)}{2 \log z\left(1-\theta_{z}\right)}\right\} \text {. }
$$

Now $\log z\left(1-\theta_{z}\right) \sim(1-\gamma) \log z$ as $z \rightarrow \infty$ and, by Lemma $2.2, \phi^{2}(z)(2 \log z)^{-1} \rightarrow$ 1. Consequently (2.4) becomes

$$
\frac{\operatorname{Var}\left(\sum_{m}^{n} I_{k}\right)}{\left(\sum_{m}^{n} E I_{k}\right)^{2}} \leq \frac{\left(1-\delta^{2}\right)^{-1 / 2}}{H_{a}^{2}(a)} \frac{M^{\prime}}{(1-\gamma)} \exp \left(\frac{M^{\prime}}{1-\gamma}\right)+o(1) \text { as } n \rightarrow \infty .
$$

From Lemma 2.1 in $[8]$ it follows that

$$
1-2 \sum_{k=1}^{\infty}\left(1-\Phi\left(1 / 2(k a)^{a / 2}\right)\right) \leq H_{a}(a) \leq 1
$$

so that $\lim _{a \rightarrow \infty} H_{a}(a)=1$. Since $a, \delta, \gamma$ and $M^{\prime}(>M)$ can be chosen arbitrarily, (2.5) becomes

$$
\frac{\operatorname{Var}\left(\sum_{m k}^{n} I_{k}\right)}{\left(\sum_{m}^{n} E I_{k}\right)^{2}} \leq M \exp (M)+o(1) \text { as } n \rightarrow \infty
$$

As noted in the introduction, condition (b) of Theorem A implies that we have a 0-1 probability for the event under study; and now equation (2.6) and Lemma (2.5) imply that the event has probability one. Q.E.D.

Corollary to Theorem A. If condition (a) of Theorem A is simplified to (a) $r(t)=1-C|t|^{\alpha}+o\left(|t|^{\alpha}\right)$ as $t \rightarrow 0$, where $0<\alpha \leq 2$ and $C>0$, then Theorem A holds with $I(\phi)=\int_{a}^{\infty} \phi(t)^{2 / x-1} \exp \left(-\phi^{2}(t) / 2\right) d t$.

3. The discrete case. In this section we give Theorem $B$ which is the discrete version of Theorem A.

Theorem B. Let $\left\{X_{n}: n \geq 1\right\}$ be a stationary Gaussian sequence with zero mean and unit variance. Suppose that the covariance function $r(n)$ satisfies the mixing condition:

$$
r(n)=O(1 / \log n) \text { as } n \rightarrow \infty .
$$

Then for any nondecreasing sequence of positive numbers $\phi(n)$ with $\phi(\infty)=\infty$, $P\left[X_{n}>\phi(n)\right.$ i.o. $]=0$ or 1 according as the sum

$$
I(\phi)=\sum_{n=n_{0}}^{\infty} \phi(n)^{-1} \exp \left(-\phi^{2}(n) / 2\right)
$$

is finite or infinite. 
Indication of the proof. When $I(\phi)<\infty$, the proof that $P\left[X_{n}>\phi(n) i_{.0}.\right]=0$ is the same as that of Qualls, Simmons and Watanabe [9]. When $I(\phi)=\infty$, we define $I_{n}$ to be the indicator function of the event $\left\{X_{n N}>\phi(n N)\right\}$ with $N$ a sufficiently large number suitably chosen. The proof of the theorem then follows on showing that the ratio $\operatorname{Var}\left(\Sigma_{k \leq n} I_{k}\right) /\left(\Sigma_{k \leq n} E\left[I_{k}\right]\right)^{2}$ is bounded. This fact is established in a manner similar to that of Theorem A. For other relevant details in this connection we refer the reader to [9].

Remarks. If $r(n) \geq 0$ for all $n$ and $(\log n) r(n) \rightarrow \infty$ as $n \rightarrow \infty$ and $\phi^{2}(n)=2 \log n$, then one can show that the ratio $\operatorname{Var}\left(\Sigma_{k \leq n} I_{k}\right) /\left(\Sigma_{k \leq n} E\left[I_{k}\right]\right)^{2} \rightarrow \infty[3]$. This shows that our Theorem B (and Theorem A) is in a certain sense the best result that Lemma 2.5 will produce.

4. Rate of growth of the number of upcrossings. In this section we shall primarily concentrate on the discrete case and develop a new line of investigation concerning the rate of growth of upcrossings. We now establish Theorem $C$ stated in $\$ 1$ whose conclusion is stronger than that of Theorem $B$ of the preceding section.

Proof of Theorem C. For simplicity, we shall furnish an outline of the proof. We use the notation of Theorem B. That $\Sigma^{\infty} E\left[I_{k}\right]=\infty$ is immediate since $E\left[I_{k}\right] \simeq$ $(2 \pi)^{-1 / 2} \phi(k)^{-1} \exp \left(-\phi^{2}(k) / 2\right)$ as $\phi(k) \rightarrow \infty$. The mixing condition that we use here is $r(n)=O\left(1 / n^{\gamma}\right)$ as $n \rightarrow \infty$. Under this condition Qualls, Simmons and Watanabe [9] have established that, for all sufficiently large $m$,

$$
\lim _{n \rightarrow \infty}\left|\sum_{m \leq i<j \leq n} \sum_{i} \operatorname{Cov}\left(I_{i}, I_{j}\right)\right|<\infty .
$$

Let $J_{n}=\Sigma^{n} I_{k}$ and consider a subsequence $\left\{J_{n_{k}}: k \geq 1\right\}$ from $\left\{J_{n}: n \geq 1\right\}$ such that $E\left[J_{n_{k}}\right] \sim k^{2}$ as $k \rightarrow \infty$. Then

$$
\begin{aligned}
P\left\{\mid J_{n_{k}}-\right. & \left.E\left[J_{n_{k}}\right] \mid>\epsilon E\left[J_{n_{k}}\right]\right\} \\
& \leq \frac{\operatorname{Var}\left(J_{n_{k}}\right)}{\epsilon^{2} E\left[J_{n_{k}}^{2}\right]}=\left(\sum_{m \leq i<j \leq n_{k}} \sum_{i} \operatorname{Cov}\left(I_{i}, J_{j}\right)+\sum^{n_{k}} V\left(I_{i}\right)\right) / \epsilon^{2} E\left[J_{n_{k}}^{2}\right]=O\left(\frac{1}{k^{2}}\right) .
\end{aligned}
$$

Therefore $\Sigma_{k \geq 1} P\left\{\left|J_{n_{k}}-E J_{n_{k}}\right| \geq \epsilon E J_{n_{k}}\right\}<\infty$, so that for each $\epsilon>0$

$$
P\left\{\left|J_{n_{k}} / E J_{n_{k}}-1\right| \geq \epsilon \text { i.o. }\right]=0 \text {. }
$$

Consequently $\lim _{k \rightarrow \infty} J_{n_{k}} / E J_{n_{k}}=1$ a.s. We show that a similar result holds for the entire sequence $\left\{J_{n}: n \geq 1\right\}$. For each $k \geq 1$, let $D_{k}=J_{n(k+1)}-J_{n_{k}}$. Then for each $\epsilon>0$, 


$$
\begin{aligned}
P\left\{D_{k}\right. & \left.\geq \epsilon E J_{n_{k}}\right\} \leq E\left[D_{k}^{2}\right] / \epsilon^{2}\left(E J_{n_{k}}\right)^{2} \\
& =\left(\sum_{n_{k} \leq i<j \leq n} \sum_{(k+1)} \operatorname{Cov}\left(I_{j}, I_{j}\right)+\sum_{n_{k^{\leq i \leq n}}(k+1)} \operatorname{Var}\left(I_{i}\right)+\left(E D_{k}\right)^{2}\right) / \epsilon^{2}\left(E J_{n_{k}}\right)^{2} \\
& =\left(1 / \epsilon^{2}\right)\left[O\left(1 / k^{4}\right)+O\left(1 / k^{2}\right)+O\left(1 / k^{3}\right)\right] \text { as } k \rightarrow \infty .
\end{aligned}
$$

Thus $\Sigma_{k \geq 1} P\left\{D_{k} \geq \epsilon E J_{n_{k}}\right\}<\infty$. This implies that $\lim _{k \rightarrow \infty} C_{k} / E J_{n_{k}}=0$ a.s.

Now for each $n \geq 1$, with $n_{k} \leq n \leq n_{k+1}$, we write

$$
J_{n} / E J_{n}=J_{n_{k}} / E J_{n_{k}} E J_{n_{k}} / E J_{n}+\left(J_{n}-J_{n_{k}}\right) / E J_{n^{*}}
$$

Since $\left|\left(J_{n}-J_{n_{k}}\right) / E J_{n}\right| \leq D_{k} / E J_{n_{k}}$ and $E J_{n_{k+1}} / E J_{n_{k}} \simeq 1$, it follows that $\lim _{n \rightarrow \infty} J_{n} / E J_{n}=1$ a.s.

Remarks. For continuous parameter processes a result analogous to that of The orem $C$ can be established as follows. Suppose we subdivide the time interval into subintervals of length $\epsilon$ each and define $I_{n}$ to be the indicator function of the event $\{X(t)>\phi(t)$ for some $t$ in $(n \epsilon,(n+1) \epsilon)\}$. Then an application of our Lemma 2.1 and a proof similar to that of Theorem $C$ will show that $\lim _{n \rightarrow \infty} \Sigma^{n} I_{k} / \Sigma^{n} E I_{k}=$ 1 a.s. Although this last result furnishes some information about the rate of growth of upcrossings of the process, the events $I_{k}$ do not truly represent upcrossings.

A more satisfactory definition of $\epsilon$-upcrossing is the one given by Pickands [5], namely $X(t)$ has an $\epsilon$-upcrossing of $\phi(t)$ at $t_{0}$ if $X\left(t_{0}\right)=\phi\left(t_{0}\right)$ and $X(t)<\phi(t)$ for all $t$ such that $t_{0}-\epsilon \leq t<t_{0}$. If we let $N(T)$ be the number of $\epsilon$-upcrossings up to the time $T$, we would like to establish that $\lim _{n \rightarrow \infty} N(T) / E N(T)=1$ a.s. Results about the tail distribution of $\max X(t)$ sharper than those of Lemma 2.1 are being investigated in order to prove this suggested rate of growth for continuous time stationary processes.

5. Acknowledgement. The authors wish to express their thanks to Donald L. Burkholder for his helpful remarks.

\section{REFERENCES}

1. W. Feller, The fundamental limit theorems in probability, Bull. Amer. Math. Soc. 51 (1945), 800-832. MR 7, 128.

2. - The general form of the so-called law of iterated logarithm, Trans. Amer. Math. Soc. 54 (1943), 373-402. MR 5, 125.

3. P. Pathak and C. Qualls, A law of iterated logarithm for stationary Gaussian processes, Technical Report \#254, University of New Mexico, Department of Math. and Statist., Albuquerque, N. Mex., 1972.

4. J. Pickands III, An iterated logarithm for the maximum in a stationary Gaussian sequence, Z. Wahrscheinlichkeitstheorie und Verw. Gebiete 12 (1969), 344-353. MR 40 \#5003.

5. - Upcrossing probabilities for stationary Gaussian processes, Trans. Amer. Math. Soc. 145 (1969), 51-73. MR $40 \# 3606$. 
6. J. Pickands III, Asymptotic properties of the maximum in a stationary Gaussian process, Trans. Amer. Math. Soc. 145 (1969), 75-86. MR 40 \#3607.

7. C. Qualls and H. Watanabe, An asymptotic 0-1 behavior of Gaussian processes, Ann. Math. Statist. 42 (1971), 2029-2035.

8. - Asymptotic properties of Gaussian processes, Ann. Math. Statist. 43 (1972), 580-596.

9. C. Qualls, G. Simmons and H. Watanabe, A note on a 0-1 law for stationary Gaussian processes, Mimeo Series \#798, Inst. of Statistics, University of North Carolina, Raleigh, N. C., 1972.

10. C. Qualls and H. Watanabe, Asymptotic properties of Gaussian random fields, Trans. Amer. Math. Soc. 177 (1973), 155-171.

11. H. Watanabe, An asymptotic property of Gaussian process, Trans. Amer. Math. Soc. 148 (1970), 233-248. MR 41 \#1134.

12. A. Zygmund, Trigonometrical series. Vol. 1, 2nd ed., Cambridge Univ. Press, New York, 1959. MR $21 \# 6498$.

DEPARTMENT OF MATHEMATICS, UNIVERSITY OF NEW MEXICO, ALBUQUERQUE, NEW MEXICO 87106 